\title{
FORMAÇÃO DE NICHOS TECNOLÓGICOS E AS ECOINOVAÇÕES: O CASO DO CIBIOGÁS NA ITAIPU BRASIL
}

\author{
Andréa Torres Barros Batinga de Mendonça \\ Doutora em Administração \\ Universidade Federal do Paraná Curitiba - PR - Brasil \\ andrea.tbbm@gmail.com https://orcid.org/0000-0002-9585-7239 \\ Sieglinde Kindl da Cunha \\ Doutora em Ciência Econômica \\ Universidade Positivo Curitiba - PR - Brasil \\ skcunha21@gmail.com http://orcid.org/0000-00031936-8422 \\ Thiago Cavalcante Nascimento \\ Doutor em Administração \\ Universidade Tecnológica Federal do Paraná Curitiba - PR - Brasil \\ thiagocn1@hotmail.com https://orcid.org/0000-0002-54528965
}

\section{RESUMO}

Este estudo tem como objetivo analisar a formação do nicho tecnológico do Centro Internacional de Estudos do Biogás na Itaipu Brasil, relacionando esse desenvolvimento ao surgimento de ecoinovações. Argumenta-se que a criação e o fortalecimento de nichos tecnológicos voltados para o desenvolvimento de ecoinovações possibilitam incrementos de qualidade de vida da população em nível local, tanto do ponto de vista econômico, quanto social, ao mesmo tempo em que contribuem para a redução de impactos ambientais. Metodologicamente, seguiu abordagem qualitativa de pesquisa, por meio de entrevistas em profundidade com gestores da Itaipu Brasil e do Cibiogás e análise de documentos. Os resultados indicam que a formação do nicho está diretamente alinhada com a literatura sobre gestão estratégia dos nichos, por meio da experimentação e nacionalização de tecnologias, voltadas para ecoinovações organizacionais, tecnológicas, sociais e institucionais que possibilitaram melhorias de qualidade de vida para comunidade e produtores no desenvolvimento dos seus negócios.

Palavras-chave: Biogás. Ecoinovação. Nichos tecnológicos.

\section{TECHNOLOGICAL NICHE FORMATION AND ECOINNOVATIONS: THE CASE OF CIBIOGAS IN ITAIPU BRASIL}

\begin{abstract}
This study aims to analyze the formation of the technological niche of the International Center for Biogas Studies at Itaipu Brazil, relating this development to the appearance of eco-innovations. It argues that the creation and strengthening of technological niches aimed at the development of ecoinnovations allow for increases in the quality of life of the population at the local level both economically and socially, while at the same time contributing to the reduction of environmental impacts. Methodologically, he followed a qualitative research approach, through in-depth interviews with Itaipu Brasil and Cibiogás managers and document analysis. The results indicate that the niche formation is directly aligned with the literature on niches strategy management, through the experimentation and nationalization of technologies, aimed at organizational, technological, social and institutional eco-innovations that enabled improvements in the quality of life for community and producers in the development of their busines.
\end{abstract}

Key words: Biogas. Ecoinnovation. Niches. Technological.

Data da submissão: $02 / 07 / 2019$

Data de aceite: $23 / 03 / 2020$

Revista de Gestão Social e Ambiental - RGSA, São Paulo, v. 13, n. 3, p. 79-97, set./dez. 2019. 
Formação de nichos tecnológicos e as ecoinovações: o caso do Cibiogás na Itaipu Brasil

\section{INTRODUÇÃO}

A discussão sobre o desenvolvimento econômico das sociedades e a relação com o meio ambiente têm ganhado destaque, não só em aspectos de teorias sociais e ambientais, mas também nas teorias que lidam com questões econômicas e de desenvolvimento tecnológicos.

Inicialmente, se discute a inovação na perspectiva schumpeteriana a partir do conceito de novas combinações que rompem com maneiras tradicionais de mudança e resultam em um novo bem, um novo processo produtivo, um novo mercado, uma nova forma organizacional e uma nova fonte de matéria-prima (Schumpeter, 1985). Essa lógica de inovação, evolucionária, pressupõe um caminho cumulativo de conhecimento advindo de experiências passadas e de novos conhecimentos, colocando a economia como resultante de um processo co-evolucionário entre tecnologia, empresa, estruturas industriais e instituições (Johnson, Edquist, \& Lundvall, 2003; Nelson, 2006a; 2006b).

A partir desse entendimento, novas perspectivas de estudo surgiram buscando analisar o processo de desenvolvimento tecnológico de produtos e processos com foco na mudança sistemática e como um processo de coevolução e de transição para um futuro sustentável (Geels, 2006; Kivimaa et al, 2019; Markad, Raven, \& Truffer, 2012). Nesse processo, destaca-se a análise multinível, ressaltando, principalmente, processos de influência em diferentes direções a partir de três níveis de estruturação e estabilidade (Geels, 2002; 2004; 2006; Wittmayer et al, 2017) a saber, o nível de nicho, onde surgem as inovações radicais a partir de pesquisas, experimentação e relacionamento entre agentes do sistema de inovação; o nível meso, dos regimes sociotécnicos; e o nível macro, dos ambientes e das mudanças estruturais da sociedade como um todo.

Entende-se ainda, que os arranjos existentes de produção e consumo desempenham um papel central na definição de como novas configurações mais sustentáveis emergem e se instalam quando se trata de transição para a sustentabilidade e que os estudos nessa área são interdisciplinares focados nas mudanças estruturais dos sistemas sociais (Mylan et al, 2019; Wittmayer et al, 2017). Porém, a transição para a sustentabilidade requer mudanças nos regimes que, muitas vezes, resultam em resistência das organizações e regimes anteriores já estabelecidos, principalmente pela percepção de ameaça aos lucros e estabilidade e por preocupações com a viabilidade tecnológica e custos sociais associados à mudança (Hess, 2016).

Como foco desse trabalho, destaca-se a análise dos nichos tecnológicos e seu processo de formação. A análise dos nichos parte da concepção da Gestão Estratégica dos Nichos (Strategic Niche Management - SNM), uma técnica que foca no estudo histórico de casos de sucessos e fracassos dos experimentos com inovações radicais sustentáveis, bem como a utilização desses casos como exemplos de formulação e tomada de decisão sobre políticas (Caniëls \& Romijn, 2008; Lopolito, Morone \& Sisto, 2011; Raven, 2005).

Como resultado do processo de formação dos nichos tecnológicos, a inovação voltada à sustentabilidade assume posto e tem-se apresentado como tema emergente que busca relacionar o desenvolvimento tecnológico, o relacionamento interorganizacional e a sustentabilidade. Incluem-se os aspectos institucionais e técnicos no processo de coevolução para o alcance de mudanças sistêmicas (Kemp \& Rotmans, 2010; Markad et al, 2012).

Assim, discute-se o conceito da ecoinovação como a aproximação entre o desenvolvimento tecnológico, a inovação e os pilares da sustentabilidade apoiado no avanço do tema desde o trabalho seminal de Fussler e James (1996) e passando pela discussão de diversos autores que se voltaram ao estudo dessa temática (Andersen, 2008; 2006; Arundel \& Kemp, 2009; Carrillo-Hermosilla, Gonzalez \& Konnola 2009; Foxon \& Andersen, 2009; Kemp \& Foxon, 2007; Reid \& Miedzinski, 2008; Rennings, 1998; Tamayo-Orbegozo, Vicente-Molina \& Villarreal-Larrinaga, 2017; Xavier, Naveiro, Aoussat \& Reyes, 2018).

A relevância da compreensão da dinâmica de nichos tecnológicos não se limita a discussões sobre coevolução e transição tecnológica (Geels, 2006; Kivimaa et al, 2019; Markad, Raven, \& Truffer, 2012), mas também envolve a criação de uma cadeia de suprimentos que envolva fornecedores de peças, prestadores de serviço e o fortalecimento de atores em determinados 
segmentos da indústria nacional capazes de adotar, aprimorar e desenvolver tecnologias (Mendonça, Cunha \& Nascimento, 2018). Assim, a importância de estudar nichos tecnológicos emerge de maneira mais acentuada no contexto das ecoinovações, tendo em vista a emergência do tema, no sentido de contribuir para o desenvolvimento de soluções capazes de atender as necessidades de desenvolvimento econômico ao mesmo tempo em que criam padrões sociais e ambientais mais adequados. Desta forma, o objetivo deste estudo é analisar a formação do nicho tecnológico do Cibiogás na Itaipu Brasil, relacionando esse desenvolvimento ao surgimento de ecoinovações.

A representatividade da Itaipu, nacional e internacionalmente, em termos de capacidade produtiva foi fator chave para a escolha do objeto de estudo, sendo esta a segunda maior usina do mundo em termos de capacidade instalada e detentora do recorde mundial de geração de energia (Itaipu, 2018). O Cibiogás, criado oficialmente em 2012, se destaca nesse estudo por atribuir em sua missão e visão o objetivo de "[...]desenvolver o produto Biogás sob os aspectos econômico, ambiental e social[...]", proporcionando "[...]às comunidades internacionais acesso a dados, informações e orientação técnica relativos ao Biogás e outras fontes de energias renováveis" (Itaipu, 2012, p. 23).

Para além desta introdução, apresenta-se a base teórica que fundamentou o desenvolvimento da pesquisa, abordando o processo de formação dos nichos tecnológicos e suas principais características e as tipologias de ecoinovações presentes na literatura. Em seguida, discutem-se os procedimentos metodológicos, a análise dos principais achados e, posteriormente, as considerações finais.

\section{NICHOS TECNOLÓGICOS E SEU PROCESSOS DE FORMAÇÃO}

Os estudos que tratam do processo de transição para a sustentabilidade reconhecem que os arranjos existentes de produção e consumo desempenham um papel central de como novas configurações mais sustentáveis emergem e se instalam (Mylan et al, 2019). Entende-se ainda, que o foco dos estudos sobre transição está na dinâmica e na governança das transições históricas e contemporâneas de sustentabilidade e que a transformação do sistema sociotécnico dá-se pelas mudanças nas relações entre diversos atores, infraestrutura e tecnologias (Kivimaa et al, 2019; Wittmayer et al, 2017).

Nessa perspectiva, propõe-se que a SNM seja entendida como a criação, o desenvolvimento e controle passo a passo de espaços protegidos para o desenvolvimento e uso de tecnologias promissoras por experimentação, com o objetivo de: 1) Aprender sobre o desejo por novas tecnologias; e 2) A melhoria de desenvolvimento e da taxa de aplicação de novas tecnologias (Kemp, Schot \& Hoogma, 1998).

Entende-se aqui que os nichos tecnológicos são espaços protegidos propícios para experimentação e desenvolvimento de inovações radicais por meio da relação dos agentes (Caniëls \& Romijn, 2008; Hermans, Apeldoorn \& Kok, 2012; Kemp et al, 1998; Lopolito et al, 2011; Schot \& Geels, 2008).

Os espaços protegidos surgem a partir de mecanismos como isenções fiscais do governo, compromissos de $\mathrm{P} \& \mathrm{D}$ entre empresas ou disposição dos futuros adotantes em participar nos testes de maneira não remunerada. Dessa forma, a proteção cria um tipo de abrigo em que vários indivíduos e grupos podem se tornar participantes engajados no processo de inovação sem se tornarem sujeitos imediatos da pressão do mercado (Caniëls \& Romijn, 2008).

Os nichos capturam múltiplos domínios sociais, políticos e culturais, pelos quais reproduzem rotinas, interesses e investimentos estabelecidos, trabalhando para manter a estabilidade e restringir a inovação (Mylan et al, 2019), mas desenvolvem relações que dão suporte às inovações radicais (como cadeias de fornecimento, relações usuário-produtor, etc.) por meio de experimentos sociotécnicos em que vários stakeholders colaboram e trocam informações, conhecimento e experiências (Caniëls \& Romijn, 2008; Lopolito et al, 2011). Chega um momento em que a 
tecnologia se torna complexa e as novidades precisam ser desenvolvidas não mais por uma única organização, mas por uma combinação de expertises de diferentes organizações especializadas, com recursos e conhecimentos próprios (Hermans, Stuiver, Beers \& Kok, 2013). Os nichos são também influenciados por ações governamentais que contribuem no processo de sua formação a partir de um conjunto de sucessivos experimentos (Kemp et al, 1998).

De acordo com Kemp et al (1998), Raven (2005), Laak, Raven e Verbong (2007), Schot e Geels (2008) e Lopolito et al (2011), os nichos são constituídos de três processos internos de formação: ligação de expectativas, processo de articulação, formação dos relacionamentos. O primeiro deles é a "ligação de expectativas" que faz referência à expressão dos atores das suas próprias expectativas para os demais atores do nicho (Kemp et al, 1998). As expectativas desempenham um papel importante nas fases iniciais de desenvolvimento de uma tecnologia, uma vez que promovem a legitimidade para que os atores invistam tempo e esforços em uma nova tecnologia que ainda não possui valor no mercado (Raven, 2005). A convergência nas expectativas dos atores se refere ao grau em que as suas estratégias, expectativas, crenças e práticas caminham na mesma direção (Geels \& Raven, 2006; Hermans et al, 2012).

Para Lopolito et al (2011), esse primeiro processo está relacionado ao fator "disposição" dos atores dos nichos. Esse fator se refere à convergência das expectativas dos atores a uma visão comum sobre uma nova tecnologia que, por sua vez, determina a difusão da disposição a participar ativamente no nicho de inovação. Este é o primeiro elemento-chave para o surgimento dos nichos quando os atores decidem fazer parte dos projetos de inovação com base em suas expectativas (Lopolito et al, 2011).

O processo de moldagem e convergência das expectativas acontece quando: a) Um número considerável de participantes compartilha as mesmas expectativas (convergência de expectativas); e b) As expectativas estão baseadas em resultados tangíveis obtidos pela experimentação (Laak et al, 2007; Lopolito et al, 2011). A convergência de expectativas ocorre mais facilmente quando as promessas da nova tecnologia são dignas de confiança (com suporte em fatos e testes) e específicas (com referência a problemas claramente identificados), com problemas ainda não contemplados pelas tecnologias existentes (Lopolito et al, 2011).

Um segundo mecanismo é o "processo de articulação" por meio dos experimentos que levam a nova tecnologia a ser socialmente aceita e incluída. Esse segundo processo interno é importante, pois dentre outros benefícios, leva à articulação de: aspectos técnicos e de especificações do projeto, políticas governamentais, significados culturais e psicológicos, mercado de quem é o produtor da nova tecnologia e quais são as necessidades dos consumidores, redes de produção, relacionamentos de infraestrutura e manutenção, efeitos sociais e ambientais (Kemp et al, 1998).

O desenvolvimento dos nichos é influenciado ainda pela "formação dos relacionamentos", o terceiro processo interno, que está relacionado ao envolvimento de novos atores no assunto e nas atividades. Nesse processo estão presentes os atores afetados pelos resultados da tecnologia, ou organizações, como grupos de cidadãos e grupos ambientais (Kemp et al, 1998; Raven, 2005).

O relacionamento é uma dimensão que merece atenção particular, pois é crítica para a experimentação, o aprendizado e a formação e convergência das expectativas sobre a nova tecnologia entre as diferentes partes. Isto porque são os atores que sustentam o desenvolvimento, carregam consigo as expectativas e articulam novos requerimentos e demandas (Caniëls \& Romijn, 2008; Raven, 2005).

O desenvolvimento e a implementação da inovação é amplamente um processo social em que se pode observar, por exemplo, que a interação mais próxima entre os atores é essencial porque elementos tácitos, informais e não codificados no novo conhecimento apenas podem ser absorvidos e compartilhados por mecanismos de comunicação intensiva e processo de learning by doing (Caniëls \& Romijn, 2008).

Esse processo interno de formação das relações requer um real processo de relacionamento entre os atores no sentido de alcançar um adequado nível de poder. Assim, a existência de atores 
poderosos no nicho se torna um elemento crucial para o seu desenvolvimento. Esse ator poderoso pode ser qualquer stakeholder que traga valores adicionais para as relações: uma empresa que processa um número considerável de recursos, uma instituição pública que processa informações relevantes ou uma instituição privada interessada em investir em inovações (Lopolito et al, 2011).

No entanto, é importante ressaltar que mesmo um ator muito poderoso não tem todos os recursos requeridos para incitar o desenvolvimento do nicho. Nesse sentido, no contexto da transição, o poder deve ser visto como um conceito de dupla face: ao mesmo tempo é um recurso individual e de relacionamento. O poder possuído por um ator individual deriva dos recursos estratégicos que ele possui, mas a influência exercida por meio desses recursos depende da estrutura de relacionamentos em que eles operam. Como consequência, o processo de criação do nicho é influenciado pela presença e distribuição de poder dentro da rede de atores locais (Lopolito et al, 2011).

Raven (2005), Laak et al (2007) e Schot e Geels (2008) acrescentam a essas dimensões o processo de aprendizagem como o fator central para a introdução de novas tecnologias na sociedade. Esse processo de aprendizagem possibilita o ajustamento da tecnologia e/ou da sociedade para melhoria das chances de sucesso da difusão da inovação. O processo é considerado bom quando é amplo, com foco não somente na otimização tecnoeconômica, mas também no alinhamento entre o técnico e o social (projetos técnicos, infraestrutura, preferências dos usuários, significados normativos e culturais), e quando é reflexivo, havendo assim uma atenção para os questionamentos adjacentes, como valores sociais, e a vontade de mudar o curso se a tecnologia não atender a esses questionamentos (Laak et al, 2007).

Para Schot e Geels (2008) a gestão estratégica do nicho (SNM) tem identificado e investigado empiricamente importantes mecanismos internos dos nichos em trajetórias de inovações sustentáveis. No entanto, na medida em que as pesquisas de SNM trazem evidências de que existe a correlação entre os projetos de experimentos e os resultados em termos de desenvolvimento de nichos tecnológicos e de mercado, fica claro que os fatores de desenvolvimento internos não são os únicos que importam. Assim, os fatores externos desempenham um papel crucial na transformação das inovações dos nichos em novos regimes, colocando a necessidade de pesquisas que relacionem os processos internos e externos, possibilitadas pela perspectiva multinível, principalmente porque as inovações radicais enfrentam duros embates com os regimes já estabelecidos e que operam para manter o status quo e restringem a mudanças de trajetórias (Schot \& Geels, 2008; Hess, 2016; Mylan et al, 2019).

Mylan et al. (2019) destacam ainda os diversos estudos empíricos que mostram o papel dos processos de aprendizagem, desenvolvimento de redes sociais e atores intermediários no surgimento dos nichos de inovações radicais, principalmente em estudos nas áreas de eletricidade e mobilidade, tais como carros elétricos e biocombustíveis, tais como Kemp et al. (2018), Geels e Ravens (2006), Laak et al. (2007) e Schot e Geels (2008).

A discussão que insere o contexto ambiental na perspectiva multinível da inovação, no seu sentido econômico e sociológico, tem se apresentado como uma questão crítica para a formação de políticas, pois coloca a tecnologia tanto como causa dos problemas ambientais, quanto como uma maneira de reduzir a pegada ecológica das atividades humanas. Assim, enfatiza-se a importância da inovação tecnológica para o desenvolvimento econômico e a inovação ambiental, incluindo o contexto institucional e os processos em um quadro em que o foco correto está na coevolução de inovações técnicas e institucionais, e no processo de transição para o alcance de mudanças sistêmicas, por meio de padrões de produção e consumo mais sustentáveis (Berkhout, 2002)Kemp \& Rotmans, 2010; Markad et al, 2012; Safarzynska, Frenken \& Van Den Bergh, 2012).

A pesquisa sobre a transição para a inovação ambiental foca no papel dos nichos como locais propícios ao surgimento de inovações radicais e de transição para um novo regime (Geels, 2002; 2004; 2006; 2011; Geels \& Kemp, 2007; Geels \& Schot, 2007). Contudo, há uma necessidade em se investigar os diferentes papéis e funções dos atores no seu desempenho como colaboradores no processo de inovação, não somente de práticas, mas possibilitando mudanças no 
Formação de nichos tecnológicos e as ecoinovações: o caso do Cibiogás na Itaipu Brasil

contexto institucional (Hermans et al, 2012; Hermans et al, 2013; Smith \& Raven, 2012). Assim, se discute na seção que se segue, as tipologias de ecoinovação levantadas para estudo e identificação daquelas praticadas no projeto em análise no nicho tecnológico desse trabalho.

\section{TIPOLOGIA DA ECOINOVAÇÃO}

$\mathrm{Na}$ década de 1970, o fato de que o desenvolvimento industrial havia produzido efeitos negativos nos sistemas de suporte à vida ganhou maior repercussão, gerando diversas discussões sobre os impactos dessa atividade em termos de poluição em larga escala, deterioração do ambiente natural e incremento de desigualdades econômicas e sociais (Elzen \& Wieczorek, 2005; Hoogma, Weber \& Elzen, 2010).

Essas constatações estimularam o debate sobre o possível conflito envolvendo os interesses econômicos e as necessidades ecológicas necessárias para perpetuação das diversas espécies que vivem nos diversos ecossistemas, além de promover alinhamento com o conceito de desenvolvimento sustentável do Relatório de Brundtland de 1987, "Nosso Futuro Comum" (Blackburn, 2007). É nesta perspectiva que Markad et al (2012) argumentam que os processos de transição sociotécnica, sistemas de inovação e emergência das tecnologias sustentáveis ganharam atenção nos 15 anos anteriores à publicação do seu trabalho.

Seguindo essa linha de raciocínio, Hoogma et al (2010) e Berkhout (2010) deixam evidente em seus trabalhos que a ciência e a tecnologia podem ser utilizadas para atuar na resolução deste conflito, integrando instituições e atores do mercado, mas que a resistência para que tais práticas se disseminem de maneira adequada ainda são acentuadas.

A ecoinovação é entendida nesse trabalho como resultado do processo de transição, assim como propõem Bossle et al (2016). Os autores consideram que a ecoinovação como resultado alcançado pelas organizações pode ser estimulada por governos e demandada pela sociedade.

A dinâmica envolvendo os temas ganhou maior repercussão com o conceito de ecoinovação desenvolvido por Fussler e James (1996), a partir de três "estabilidades". A primeira delas, denominada estabilidade ecológica, se relaciona ao funcionamento do sistema natural, envolvendo a qualidade no fornecimento de água e ar. Por sua vez, a estabilidade de recursos se relaciona à acessibilidade dos seres humanos aos requisitos físicos e materiais mínimos e aos custos adequados para sua subsistência. Por fim, a estabilidade socioeconômica, insere a discussão de nível de empregos criminalidade, desigualdades excessivas em renda e saúde.

Predominantemente, os autores que abordam de maneira direta e indireta a relação entre inovação e meio ambiente analisam o fenômeno a partir da perspectiva evolucionária, tais como Carrillo-Hermosilla et al (2009), Foxon e Andersen (2009), Arundel e Kemp (2009), Reid e Miedzinski (2008), Kemp e Foxon (2007) e Rennings (1998). Bossle et al (2016) acrescentam que a maioria dos estudos adota a definição da Organização para a Cooperação e Desenvolvimento Econômico (OCDE) de inovação que traz benefícios ao meio ambiente. Nesse sentido ainda, a literatura fornece diversos modelos que auxiliam no entendimento sobre a dinâmica da ecoinovação para compreensão das organizações, para estruturação de processos sustentáveis e para pesquisadores, no sentido de aprofundar o desenvolvimento sustentável (Tamayo-Orbegozo et al, 2017; Xavier et al, 2018).

Dentre os principais trabalhos sobre o tema, destaca-se a visão de Rennings (1998) e sua proposição taxinômica de classificação das ecoinovações. Para o autor, esse tipo de inovação pode ser de natureza tecnológica, quando relacionadas a tecnologias preventivas e curativas; de natureza organizacional, quando relacionadas à mudança nos instrumentos de gestão; de natureza social, voltada a mudanças no comportamento e estilo de vida dos consumidores; e de natureza institucional, relacionada à criação de redes locais e agências, governança global e comércio internacional.

Outra proposta de classificação destacada na literatura foi desenvolvida por Kemp e Foxon (2007), tomando como base a percepção de que a ecoinovação não deve envolver apenas a redução

Revista de Gestão Social e Ambiental - RGSA, São Paulo, v. 13, n. 3, p. 79-97, set./dez. 2019. 
de impactos ambientais, contemplando, também a produção, aplicação ou exploração de um bem, serviço, processo produtivo, estrutura organizacional ou modelo de gestão para uma empresa. Nessa perspectiva, os autores propõem que a classificação deste tipo de inovação deve envolver a segmentação em tecnologias ambientais, inovações organizacionais, inovações em produtos ou serviços e, por fim, desenvolvimento de sistemas verdes.

Seguindo posicionamento semelhante ao de Kemp e Foxon (2007), Andersen (2008), argumenta que a ecoinovação corresponde a uma inovação com capacidade de gerar rentabilidade verde no mercado, colocando a firma no centro da análise do desenvolvimento de soluções que integrem problemas ambientais e o processo econômico.

Para tanto, o autor propõe uma taxonomia refletindo os diferentes papéis de um mercado verde, sugerindo: ecoinovações add-on relacionadas a produtos, tecnologias e serviços que melhoram o desempenho ambiental dos consumidores; ecoinovações integradas que fazem tanto o processo produtivo quanto o produto mais ecoeficiente ("limpo") do que seus similares; ecoinovações de produtos alternativos que representam uma nova trajetória tecnológica de inovações radicais, não sendo inovações mais limpas que seus produtos similares, mas oferecendo uma solução diferente e mais ambientalmente eficaz em comparação aos produtos existentes; ecoinovações macro-organizacionais ou novas estruturas organizacionais que representam uma nova forma ecoeficiente de organizar a sociedade, ou seja, novas formas de organização da produção e consumo em um nível mais sistemático; ecoinovações de propósito geral, que se referem a tecnologias de propósitos gerais que afetam a economia e o processo de inovação.

Dentre os modelos de classificação, Carrilho-Hermosilla et al (2009) partem da concepção de que a ecoinovação corresponde a um processo de mudança sistêmica, seja ela tecnológica e/ou social, que resulta em uma aplicação prática que melhora o desempenho ambiental. Nesse sentido, os autores segmentam estas ecoinovações em quatro dimensões: dimensão de design relacionada a fatores como adição de componentes e otimização e mudanças (redesenho) no sistema; dimensão do usuário relacionada à difusão da inovação e ao envolvimento do usuário no desenvolvimento do produto ou serviço e na mudança de comportamento; produto/serviço que envolve a criação de valor em produtos, serviços e processos e a mudança na rede de valor, e; papel da governança que se refere às novas soluções institucionais e organizacionais para resolver conflitos sobre recursos ambientais, tanto no setor público quanto no privado e está relacionada às mudanças sistêmicas.

Tamayo-Orbegozo et al (2017) propõem um modelo para complementar as análises lineares anteriores a partir de três níveis que integram fatores internos e externos no processo de ecoinovação e as relações entre os agentes. Para tanto, os autores entendem que o nível macro pressupõe um ambiente mais amplo em que está a população e os regulamentos entendidos como pontos de partida para o processo de ecoinovação, vistos como direcionadores das ações das organizações. No nível meso, encontram-se as tendências de mercado e indústria em que se encontra a organização que busca desenvolver ecoinovações para se diferenciar. E por fim, o nível micro aborda os fatores internos como recursos e habilidades, capacidades relacionais e recursos humanos capacitados para a ecoinovação (Tamayo-Orbegozo et al, 2017).

É interessante observar que, de maneira direta ou indireta, todas as tipologias envolvem mudanças em produtos ou serviços voltados a redução do impacto ambiental e redução no uso de recursos, visto as tipologias que falam em ecoinovações de natureza tecnológicas (Andersen, 2008; Kemp \& Foxon, 2007; Rennings, 1998). Outro ponto em comum diz respeito ao olhar sobre os sistemas de inovação quando as tipologias abordam que as ecoinovações envolvem inovações que extrapolam o sentido de tecnologias de produtos e serviços, mas incluem também aspectos institucionais, de governança e consumo (Andersen, 2008; Carrilho-Hermosilla et al (2009; Kemp \& Foxon, 2007; Rennings, 1998).

Destaca-se ainda que algumas tipologias, tais como de Reid e Miedzinski (2008) e,TamayoOrbegozo et al (2017), consideram três níveis para análise, o que vai ao encontro da perspectiva multinível discutida nesse trabalho. Entende-se aqui que esses modelos servem como uma descrição 
Formação de nichos tecnológicos e as ecoinovações: o caso do Cibiogás na Itaipu Brasil

ou orientação de práticas de ecoinovação (Xavier et al, 2018) e foram utilizados como base para análise e classificação dos resultados da pesquisa.

\section{PROCEDIMENTOS METODOLÓGICOS}

Com o objetivo de analisar o processo de formação no nicho tecnológico do Centro Internacional de Estudos do Biogás (Cibiogás) na Itaipu Brasil, relacionando seu desenvolvimento ao surgimento de ecoinovações, realizou-se pesquisa de estratégia qualitativa, principalmente pela característica das informações necessárias para entender o fenômeno de formação do nicho em seu contexto (Walliman, 2006). Além disso, a escolha dessa estratégia justifica-se pela busca dos significados e interpretação dos dados pelos envolvidos no processo e as visões que compartilham a partir de pesquisa in loco (Denzin \& Lincoln, 2006; Merriam, 2009).

Foi realizado um estudo de caso descritivo com vista a analisar causas e consequências de fenômenos que não são passíveis de análise quantitativa, buscando estabelecer o que é normal (Richardson, 2007; Walliman, 2006), a partir da análise da formação dos nichos tecnológicos, as relações multiníveis e as ecoinovações encontradas nos projetos da Itaipu Brasil.

Escolheu-se a Itaipu Brasil, primeiramente, pela relevância da fonte hidráulica de geração de energia, responsável por cerca de 65\% de geração no Brasil (Empresa de Pesquisa Energética, 2019) e por também ser considerada uma fonte renovável de energia, mas, principalmente, pela representatividade nacional e internacional da Itaipu, sendo a maior do mundo em geração de energia, responsável por 15\% da energia consumida no Brasil (Itaipu, 2020).

Para se chegar especificamente no programa Cibiogás, foram realizadas duas entrevistas. Uma, com o Assessor de Planejamento Estratégico, e, outra, com o Coordenador do Sistema de Gestão da Sustentabilidade (SGS) da Itaipu Brasil. Essas entrevistas foram realizadas principalmente por serem esses gestores os responsáveis pela inclusão e difusão da sustentabilidade na Itaipu Brasil, um que trata, de maneira transversal, o tema no SGS e o outro na perspectiva estratégica. A partir dessas entrevistas, que duraram respectivamente $2 \mathrm{~h} 53 \mathrm{~m}$ e $2 \mathrm{~h}$, chegou-se à indicação do Programa do Centro Internacional de Estudos do Biogás - Cibiogás como um dos programas de destaque da Itaipu voltado para o desenvolvimento de inovações com forte apelo de sustentabilidade.

A partir de então, entrou-se em contato com o Diretor do Cibiogás, que, por questões de incompatibilidade de agenda, indicou os demais membros da equipe que responderiam a pesquisa, entre eles o Gerente de Relações Institucionais e Internacionais que é um dos funcionários mais antigos, estando no Ciobiogás desde a sua concepção. Foram realizadas assim, duas entrevistas no próprio laboratório do Cibiogás na Itaipu, em Foz do Iguaçu. A primeira entrevista foi com o coordenador do laboratório do Biogás, que durou $1 \mathrm{~h} 16 \mathrm{~m}$, e uma com o Gerente de Relações Institucionais e Internacionais do Cibiogás, que durou $42 \mathrm{~m}$. Foi importante estar no próprio local de trabalho, pois foi possível observar as instalações e como se dá o trabalho de estudo do Centro. Nesse momento, dois pesquisadores acompanharam a visita, mostraram o laboratório e explicaram como se dá o processo de análise dos materiais e a determinação da capacidade de geração de energia, a partir do biogás, e contaram das suas experiências.

Além das entrevistas foi possível ter acesso ao Projeto de Concretização e Estatuto do Centro que conta sobre a sua estruturação, principais agentes parceiros e a história de formalização do centro dentro da própria Itaipu e sua atuação internacional.

Após a coleta de dados, foi utilizada a análise de conteúdo, com base em conceitos e instruções de Bardin (2011), com o objetivo de descrever melhor os conteúdos das mensagens contidas nas entrevistas, nas observações de campo e nos documentos analisados. Especificamente sobre o Cibiogás, no decorrer da análise, surgiram algumas categorias de análise no software Atlas Ti que facilitaram o entendimento da formação do nicho e dos tipos de ecoinovações: "O Programa", "Resultados e Mudanças", "Trajetória Tecnológica", "O Nicho", "Barreiras", "Facilitadores" e "Planos Futuros". Estas categorias foram analisadas de maneira conjunta, com o 
objetivo de explorar a formação do nicho tecnológico do programa em estudo, destacando o histórico de desenvolvimento, o fortalecimento institucional e a formação de uma rede de agentes responsáveis pela formação do nicho. Assim, as presentes categorias se manifestam ao longo de todo o relato.

A partir desses procedimentos, chegou-se à descrição da formação das relações do nicho tecnológico e das ecoinovações desenvolvidas que serão discutidas na seção a seguir.

\section{A FORMAÇÃO DO NICHO TECNOLÓGICO DO PROGRAMA CIBIOGÁS}

O nicho tecnológico em destaque foi desenvolvido no Parque Tecnológico Itaipu (PTI), um espaço privado voltado para a formação de parcerias entre diferentes agentes com vistas a experimentar e desenvolver inovações radicais (Kemp et al, 1998; Safarzynska, Frenken \& Van Den Berg, 2012). Esse espaço surgiu em 2003, principalmente, por um incentivo da própria organização para o desenvolvimento de novas tecnologias (Caniëls \& Romijn, 2008) e difusão de conhecimento, integrando empresas, centros de pesquisa, laboratórios e instituições de ensino, não só do Brasil, mas de outros países também. Dessa forma, o que é desenvolvido e difundido por beneficiar a sociedade como um todo.

Com mudanças introduzidas na missão da Itaipu que possibilitaram, em 2003, incluir as preocupações com a sustentabilidade ambiental, social e econômica, foram realizadas diversas ações e mudanças na estrutura, nos projetos e programas da Itaipu Binacional, especificamente na margem brasileira. Uma dessas mudanças ocorreu em 2008, no organograma da organização, que passou a incluir a Assessoria de Energias Renováveis. Essa assessoria propôs o programa Plataforma de Energias Renováveis como um dos seus trabalhos, buscando, por meio de estudos, demonstrar a viabilidade técnica, econômica e ambiental das fontes renováveis de energia a partir de projetos acompanhados pelo PTI (Itaipu, 2012).

$\mathrm{O}$ avanço do projeto se deu com a parceria firmada entre a Organização das Nações Unidas para o Desenvolvimento Industrial (Onudi), Eletrobras e Itaipu, estabelecendo a implantação do Observatório de Energias Renováveis, projeto que envolvia toda a América Latina e o Caribe. Esse projeto tem por objetivo manter o fluxo de informações e conhecimento sobre o tema das Energias Renováveis, além de divulgar o desenvolvimento de tecnologias e metodologias que aplicassem a energia no desenvolvimento.

Dessa forma, uma das ações foi a criação do Laboratório do Biogás, cuja metodologia estava baseada na experiência previamente desenvolvida na Universidade da Terra em Viena (Áustria), culminando na criação do Centro de Estudos do Biogás para que este fosse o responsável pela gestão das ações e demandas que surgissem nessa área para Itaipu e Fundação PTI (Itaipu, 2012).

Com relação aos serviços, o Centro tem como competência a prestação dos seguintes serviços: desenvolvimento tecnológico e difusão do conhecimento em Energias renováveis - biogás e plataforma tecnológica - unidades de demonstração. Serão discutidos adiante os projetos já colocados em prática pelo centro.

O Coordenador do Laboratório é enfático ao dizer que o Centro "Vende conhecimento, vende tecnologia, vende curso, vende ensaio, vende consultoria e assim por diante”, mas o Gerente de Relações Institucionais e Internacionais completa que eles podem ainda ser contratados para executar e coordenar atividades. Segundo ele: "A gente faz estudo pelo laboratório, mas a gente também pode executar projetos".

O Gerente de Relações Institucionais e Internacionais destaca também como iniciativa interna, a instalação de um biodigestor no PTI para aproveitar restos de comida do restaurante para gerar energia. No entanto, o entrevistado não sabe se ainda está em funcionamento, relatando que, na época de sua instalação, o equipamento funcionava adequadamente.

Em 2011, na Conferência Mundial de Energia, foi entregue pelo Diretor Geral da Itaipu Brasil, José Miguel Samek, ao Diretor Geral da Onudi, uma carta expondo as razões para a implantação de um Centro Internacional de Energias Renováveis com ênfase no Biogás no PTI. O 
centro foi estruturado com base nas normas de organização de Centros Internacionais de Tecnologia (ITC) da Onudi. A carta foi bem recebida pelo Diretor Geral da Onudi e a apresentação final do Centro foi feita na Rio+20, em 2012 (Itaipu, 2012).

Segundo o Gerente de Relações Institucionais e Internacionais do Centro, na Rio+20 foi apresentada a proposta de criação do Centro Internacional de Energia Renováveis em um documento como protocolo de intenção do desenvolvimento de estudos do Cibiogás. Este documento foi assinado por diversas autoridades presentes.

Ele foi assinado pela Itaipu Binacional, pela Eletrobras, inclusive o presidente da Eletrobras, [...] o diretor geral da época da Onudi, que a sede fica em Viena, a Olade que é a organização latino-americana de energia, a Embrapa, o Ceap não assinou, a Copel, a Cepel, centro de estudos de energia elétrica da Eletrobras, assinou a Fiep, a Faep não assinou, a Ocepar que é a organização das cooperativas do Paraná, o Cepgás que o Centro de Pesquisa do gás da Petrobras, que fica em Natal, o Senai nacional e do Rio Grande do Norte, o PTI, o Ministro Lobão e o Ministro de Desenvolvimento Agrário e mais duas testemunhas (Entrevista com o Gerente de Relações Institucionais e Internacionais).

As relações formadas nesse nicho tecnológico envolveram, inicialmente, a Itaipu, a Onudi e a Eletrobras, para a formação de um Observatório de Energias Renováveis. No entanto, o projeto cresceu até se tornar um programa ainda vinculado ao Parque Tecnológico de Itaipu e, posteriormente, foi concretizado como Centro Internacional de Energias Renováveis-Biogás, reconhecida juridicamente como uma associação sem fins lucrativos.

A seguir, é possível visualizar a formação do nicho tecnológico do Cibiogás a partir dos principais parceiros identificados.

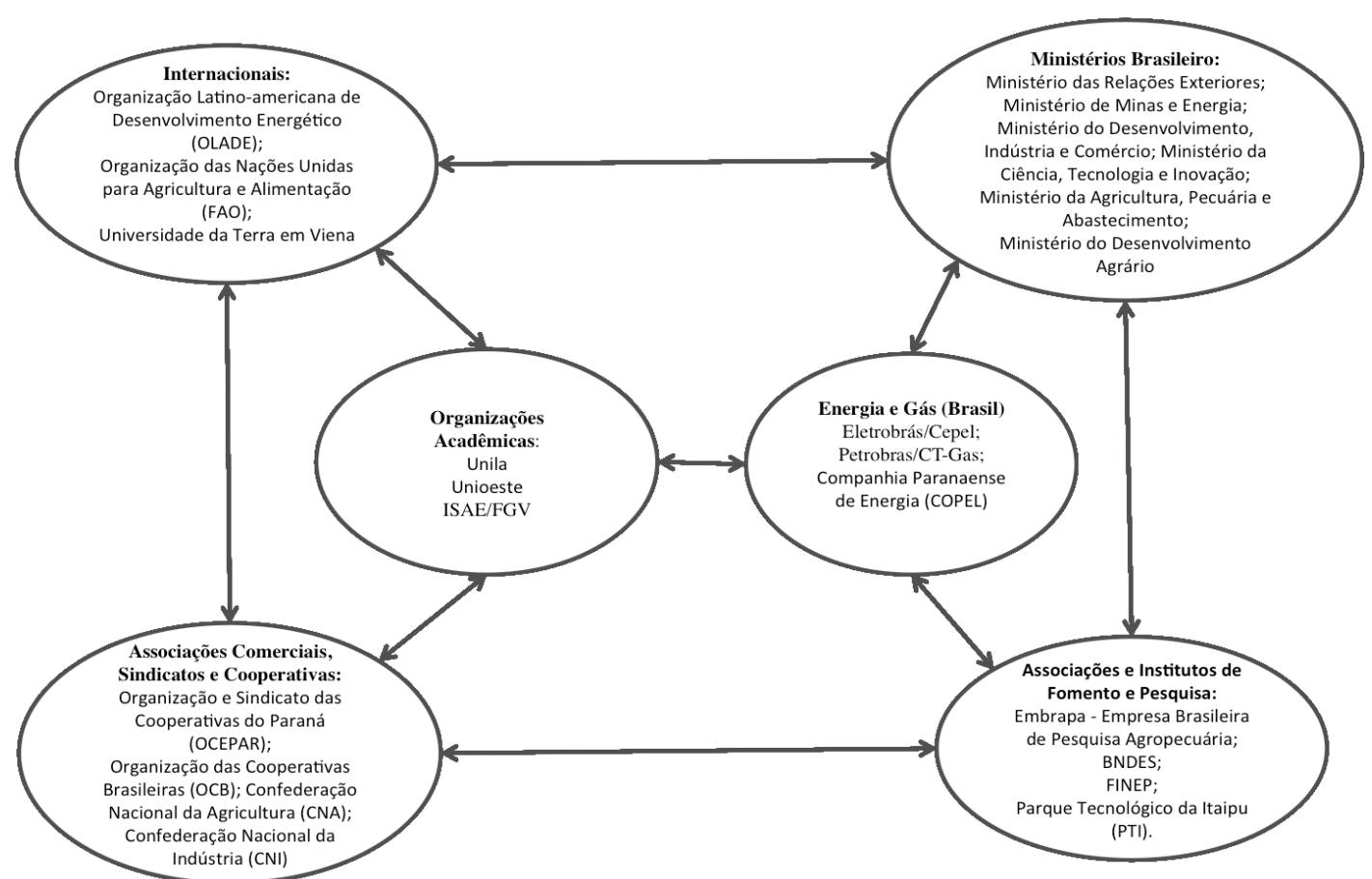

Figura 1 - Os agentes do Nicho Tecnológico do Programa Cibiogás

Fonte: Elaboração própria

Percebe-se que poucas instituições de ensino superior se inseriram no programa como agentes constituintes do nicho. Dentre as três organizações presentes, destaca-se a presença da Universidade Federal da Integração Latino-Americana (Unila) que se encontra situada nas instalações do Parque Tecnológico de Itaipu, ou seja, compartilha laboratórios e está sediada 
próxima ao Cibiogás e a Universidade Estadual do Oeste do Paraná (Unioeste) que também atua na região. Assim, o programa buscou fortalecer sua atuação com instituições de ensino locais, como uma estratégia de desenvolvimento regional.

A importância das parcerias foi destaque nas duas entrevistas com os gestores do Cibiogás. Merece destaque inicial a troca de conhecimento entre o laboratório e a Universidade de Viena. O Coordenador do Laboratório evidencia a fase inicial de desenvolvimento do programa, quando o laboratório precisou entender melhor como os ensaios com o biogás eram conduzidos.

O primeiro resultado a observar diz respeito às unidades de demonstração, que são projetos dos técnicos do laboratório de biogás, parceiros, como as prefeituras, e, principalmente, os criadores das cidades de Foz do Iguaçu e região. Em 2012, chegou-se ao total de 8 unidades de demonstração instaladas e em andamento. Em 2019, já conta com 11 unidades de produção de biogás no Brasil (Cibiogás, 2019).

No entanto, segundo destaca o Gerente de Relações Institucionais e Internacionais, o Centro contava, em 2014, também com uma unidade de demonstração internacional em San José, no Uruguai, que era uma replicação das condições estudadas e instaladas no Condomínio de Ajuricaba, que discutiremos mais à frente.

O funcionamento dos estudos e, principalmente, do laboratório se baseia em pegar os dejetos dessas comunidades, seja de suínos, bovinos, aves ou de humanos, para testar e verificar qual o potencial de geração de energia a partir do tratamento desses dejetos. Sendo assim, cada projeto é único, pois as características dos dejetos mudam dependendo, muitas vezes, da forma de alimentação dos animais das fazendas, como explicam os gestores e a técnica do laboratório empregada.

A seguir, destacam-se alguns quesitos relevantes do contexto de aplicação de três dessas unidades de demonstração, conforme explicação do Gerente de Relações Institucionais e Internacionais. A primeira unidade de demonstração destacada pelo gerente é a Colombari, uma fazenda que engorda suínos para abatedouros e que começou tratando os dejetos de três mil porcos em fase de abate. A instalação do biodigestor resolveu os problemas ambientais gerados pelos dejetos, conseguindo com isso, a autorização para criar mais 2.000 porcos.

[...] tem uma granja chamada granja Colombari que é uma pequena fazenda de uma pessoa só, de uma família que produz, que engorda suínos para levar para os abatedouros, então são suínos em terminação [...]. Em Colombari tinham 3.000 suínos antes e estava com muitos problemas sobre o destino dos dejetos desses suínos que estava tendo até problemas ambientais com o Iapar, estava recebendo multas, porque não estava conseguindo dar terminação aos dejetos desses 3.000 suínos. E o Colombari gastava 3.000 reais por mês só em conta de luz. Porque ele planta o milho, ele processa o milho para ele alimentar esse... então ele tem uma pequena indústria, para ele alimentar esses suínos. [...] hoje ele capta esses dejetos dos suínos dele, coloca um biodigestor e ele gera, além de biofertilizante que ele usa na agricultura dele, ele gera energia elétrica, ele é autossustentável em energia elétrica. Ele não gasta mais nenhum centavo em energia elétrica para a Copel. A energia elétrica dele é gerada na própria propriedade e ela é consumida e o excesso ainda é enviada para a Copel. E ele resolveu esse problema de saneamento dele porque ele está tratando esse dejeto, então esse dejeto parou de ser um problema para o meio ambiente. O que aconteceu com isso foi que ele conseguiu autorização para ter mais 2.000 suínos, hoje ele tem 5.000. Porque ele dá um trato correto aos dejetos (Entrevista com o Gerente de Relações Institucionais e Internacionais).

A destinação do excedente de energia produzida pela Colombari é enviada para a Copel, consistindo em uma das mudanças mais significativas de atuação do Centro. Os entrevistados destacam esse fato como um ganho para a população, pois os criadores passam a acreditar na 
viabilidade do negócio e a querer realmente tratar dejetos nas suas fazendas, já que percebem que, além do ganho ambiental, eles têm um benefício financeiro.

A segunda unidade, destacada pelo Gerente, é a Cooperativa Lar, uma unidade produtora de aves. Segundo o Gerente, eles processavam cerca de 200 mil frangos por dia e mesmo por se tratar de uma cooperativa com um sistema moderno tecnologicamente, ainda tinham problemas na destinação final dos dejetos desses 200 mil frangos.

Cada parte do frango segue em uma esteira mecânica, e tudo que não serve para ser processado e vendido vira resíduo. Esse resíduo, ele passa por uma, primeira filtragem, parte dele é transformada em ração para pet, para animais domésticos, a outra parte vai para os biodigestores, eles também estavam com problemas ambientais que você imagina 200 mil frangos por dia. [...] e essa fábrica ela parava de trabalhar às 6 da tarde e voltava às 21 da noite, porque você sabe que de 6 da tarde às 9 da noite é maior demanda elétrica no Brasil. É o horário que as pessoas chegam em casa, ligam a luz, liga a TV, a geladeira, liga de tudo quando chega em casa, liga o chuveiro. Então o pico é maior de demanda de energia, a energia que é 10 vezes mais cara [...] Então essa indústria não podia trabalhar nesse período senão ficaria inviável. Não ia conseguir agregar esse valor no processo do frango [...]. Hoje ela está produzindo energia e está usando nesse horário, que, ao invés de ela pagar 10 vezes mais, ela usa a energia que ela produz. Então hoje ela funciona $24 \mathrm{~h}$ por dia, então ela gera mais empregos e polui menos ainda. É impressionante, lá tem dois biodigestores do tamanho de um campo de futebol cada um, é uma coisa impressionante. E eles ainda usam biofertilizante, eles têm o que eles chamam de floresta energética, eles plantam eucalipto lá, e esse eucalipto é usado nos processos industriais para aquecer boiler, para queimar mesmo. E eles regam os eucaliptos com biofertilizante. E eles produzem ração para pet, produzem biogás, produzem biofertilizantes, geram energia elétrica, então você imagina o impacto disso na cadeia industrial (Entrevista com o Gerente de Relações Institucionais e Internacionais).

O terceiro caso destacado pelo Gerente de Relações Institucionais e Internacionais é o Condomínio Ajuricaba, Condomínio de Energias Renováveis da Agricultura Familiar na Bacia do Rio Ajuricaba, em Marechal Cândido Rondon. Este caso foi pensado para ser conduzido em forma de condomínio por se tratar de pequenos produtores, isto é, não ficaria viável a implantação de um biodigestor individual. São 33 pequenos produtores conectados por um biogasoduto de $25 \mathrm{~km}$ de extensão que passa pelas propriedades e culmina em uma central onde o gás é distribuído para um secador de grãos comunitário que seca o milho e a soja produzidos antes de serem estocados.

[...] antes eles colocavam em caminhão, levavam para uma indústria, a indústria secava e eles traziam de volta. Então, eles gastavam diesel e tinham que pagar por esse serviço. Hoje, eles usam o secador comunitário que seca os grãos deles igualzinho, perfeito, lá com o biogás deles, inclusive (Entrevista com o Gerente de Relações Institucionais e Internacionais).

A outra parte do gás vai para um filtro e depois para um motor e então, cada produtor tem uma cota que pode usar em seus biodigestores individuais para gerar energia. $\mathrm{O}$ entrevistado ressalta alguns aspectos sociais da construção deste biogasoduto comunitário.

Então, ele é muito interessante esse Ajuricaba, porque ele tem essa conformação social. Ele envolve pessoas, 33 proprietários diferentes, ele gera energia térmica, ele gera energia elétrica, ele gera biofertilizante. Aí você conversa com as mulheres dos agricultores e a primeira coisa que eles dizem para você é que eles não têm mais vergonha de receber visita nas propriedades delas, porque não tem mau cheiro mais, e não tem mosquito mais, porque todo esse dejeto está sendo tratado. Então 
esse é um aspecto social, de saúde pública. E muitas dessas propriedades jogavam os dejetos naquelas piscinas para fazer esse tratamento, muitas vezes chovia, alagava, poluía a terra, e muitas dessas propriedades tinham poço artesiano pertinho e eles tomavam daquela água (Entrevista com o Gerente de Relações Institucionais e Internacionais).

Além dos resultados relacionados às unidades de demonstração, é importante mencionar os demais pontos positivos destacados pelos entrevistados e algumas mudanças após a concretização do Centro de Estudos. Algumas dessas mudanças fazem menção a pontos já comentados pelo Gerente entrevistado quando fala sobre as unidades de demonstração.

Os resultados socioeconômicos e ambientais dizem respeito, principalmente, a diminuição da poluição do solo e da água pelos dejetos dos animais criados nessas localidades assistidas pelo programa. Além dessa melhoria, houve benefícios sociais e econômicos para a população, como a geração própria de energia, diminuindo a compra de energia da Copel e, consequentemente, a redução dos custos. Além disso, ocorreu a melhoria da qualidade de vida com a diminuição do perigo de contaminação pelo não tratamento adequado desses dejetos. O Coordenador do Laboratório do Biogás destaca:

[...] então acho que o melhor resultado de tudo é, vamos dizer assim, a catapulta social que isso foi. De poder gerar mais qualidade de vida, mais qualidade de viver, mais conhecimento, fixação desse povo na região e renda. Emprego e renda (Entrevista com o Coordenado do Laboratório).

Com a descrição dos principais resultados e mudanças, foi possível identificar a visão dos entrevistados com relação ao momento atual do Cibiogás, que se encontra formalizado e adequado para o desenvolvimento de novas tecnologias por meio de parcerias, trocas de conhecimento, pesquisas e experimentos de novas técnicas. Nesse sentido, o Coordenador destaca a importância de aproximação com as universidades por meio de cursos de mestrado e doutorado que possibilitem aos engenheiros melhor desenvolvimento de seus projetos.

$\mathrm{Na}$ visão do Coordenador, deve-se evitar importar a tecnologia por meio da inovação. Citando um exemplo, ele destaca o fato de existirem muitos filtros, nacionais e importados, no mercado, mas que "[...] ]eu tenho que fazer o melhor filtro, com a melhor matéria-prima brasileira da forma mais barata e de mais fácil logística e distribuição pra fazer".

[...] foram agrupadas várias tecnologias de interesses isolados, entre uma fermentação, entre um processo de filtragem e tratamento, entre um processo de queima do próprio gás, um processo de ligar isso na rede e juntou em linha e montou a tecnologia de se transformar um resto de dejeto de porco em um kilowatt de energia. Então, não teve um desenvolvimento de uma tecnologia que fosse extremamente nova como o Iphone antes do Iphone existir. Um touchscreen antes dele existir. Mas então foi assim, foi uma inovação articulada né, você pega várias coisas que existem, junta todas e vira uma coisa que não existia antes (Entrevista com o Coordenador do Laboratório).

A partir das relações constituídas nesse nicho tecnológico e com base na pesquisa realizada foi possível perceber que, pelo caráter distinto do tipo de atividade e atuação de cada agente, houve um processo de combinação de experiências e conhecimentos para o desenvolvimento das tecnologias, cada um com seu know how e expertises (Caniëls, \& Romijn, 2008; Lopolito et al, 2011).

A partir da perspectiva de inovação de Schumpeter (1985), observa-se convergência entre teoria e prática, quando os entrevistados destacam a combinação de diferentes tecnologias, conhecimentos e matérias-primas para o desenvolvimento das tecnologias no Centro de Estudo, 
principalmente voltadas ao contexto nacional, já que a realidade do Brasil diferem de demais países que utilizam esta tecnologia.

Assim, o interessante nesse ponto está na nacionalização da tecnologia e o fato de os agentes se voltarem à pesquisa e ao desenvolvimento para atender as demandas locais. Vellinga (2000) destaca a importância de iniciativas locais para a transformação, principalmente no sentido do desenvolvimento de tecnologias end-of-pipe.

O fato de haver a replicação dos conceitos e das tecnologias do Centro também leva ao entendimento da experimentação nos nichos tecnológicos, como acrescenta a abordagem de Kemp et al (1998), no sentido de que nos nichos, a presença dos agentes formando relações nesses espaços protegidos facilita a experimentação das tecnologias para que aprendam e melhorem o seu desenvolvimento.

Destacam-se os três processos internos dos nichos elencados na literatura (Kemp et al., 1998; Laak et al., 2007; Lopolito et al., 2011; Raven, 2005; Schot, \& Geels, 2008). No caso da formação do nicho do Cibiogás, foi possível perceber que se desenvolveu a partir da convergência de expectativas e disposição dos agentes no desenvolvimento da tecnologia, na medida em que esses agentes foram aprendendo e entendendo mais sobre a tecnologia e investindo esforços para o avanço do conhecimento (Geels, \& Raven, 2006; Lopolito et al, 2011).

O segundo ponto do desenvolvimento do nicho percebido foi o "processo de articulação", relacionado à aceitação social da tecnologia em experimentação que leva à articulação dos agentes organizacionais e sociais, bem como da articulação do mercado (Kemp et al., 1998).

No entanto, nota-se que esse processo de articulação não está totalmente desenvolvido no sentido de que a tecnologia está somente difundida entre os agentes que participam do nicho em questão, não havendo total disseminação e articulação dos agentes, no que diz respeito ao desenvolvimento da infraestrutura de mercado.

O desenvolvimento do nicho é também influenciado pela formação dos relacionamentos, como já demonstrado na Figura 1, com os atores envolvidos no processo de experimentação e desenvolvimento da tecnologia. Esses mecanismos internos dos nichos são importantes para o processo social de inovação no sentido de que o desenvolvimento tecnológico faz parte de uma aprendizagem do tipo learn by doing, o que está em convergência com o sentido de experimentação e compartilhamento de conhecimento do nicho (Caniëls, \& Romijn, 2008; Raven, 2005).

Nesse processo de formação dos relacionamentos, destaca-se a figura de diversos atores poderosos que se caracterizam como stakeholders, com muitos recursos e poder de articulação com outros agentes (Lopolito et al, 2011). Uma vez que se analisa aqui o nicho formado a partir da iniciativa da Itaipu Brasil, essa organização é o principal agente nesse processo de relacionamentos.

Além desses fatores de formação nos nichos, o processo de aprendizagem é fator de destaque, uma vez que possibilita a introdução social da tecnologia a partir da busca de seu entendimento e da percepção da sua viabilidade econômica (Laak et al., 2007; Raven, 2005; Schot, $\&$ Geels, 2008). Nesse sentido, o que se observou, durante as entrevistas, foi o destaque dos gestores à possibilidade de aprendizado que se tem diante das relações que foram formadas, principalmente devido ao grau de conhecimento que cada ator possui na sua área de competência.

De forma complementar, o desenvolvimento da inovação nesse nicho se configura em uma perspectiva evolucionária da inovação, no sentido de envolver diferentes tecnologias, estruturas industriais e instituições, em um processo de coevolução, novas combinações e novos conhecimentos (Johnson et al., 2003; Nelson, 2006b; Schumpeter, 1985).

Dessa forma, e como primeiros resultados dessas novas combinações, tem-se a introdução no mercado nacional, e, principalmente, local, de um novo bem, quer seja a energia a partir do biogás, quer seja pelas tecnologias para sua transformação, além de um novo processo de produção, como a nova forma de gerar energia pela biomassa, a abertura de um novo mercado ainda em desenvolvimento, principalmente com relação à infraestrutura e a nova fonte de matéria-prima, neste caso a própria biomassa para a geração de energia (Schumpeter, 1985). 
A partir do processo de formação dos nichos e das inovações que são desenvolvidas, percebe-se a influência bottom up e top down de mudança no nível dos regimes sociotécnicos como forma de tentar mudar o regime em vigência (Geels, 2011; Genus, \& Coles, 2008).

O que se notou é que as mudanças iniciadas pelo nicho do Cibiogás estão concentradas em um contexto local, passando para um nível regional de atuação pelo avanço não só em melhorias end-of-pipe, mas principalmente em processos (Vellinga, 2000).

O alcance da ecoinovação não seria apenas inovações em produtos e processos voltados para a melhoria ou preservação do ambiente, mas em uma mudança sistêmica (Andersen, 2008; CarrilloHermosilla et al., 2009; Rennings, 1998).

Como resultado, foi possível identificar os tipos de ecoinovações já desenvolvidas ou em fase de desenvolvimento pelo nicho, a partir da figura 2 a seguir:

\begin{tabular}{|c|l|l|}
\hline Ecoinovações & \multicolumn{1}{|c|}{ O que é? } & \multicolumn{1}{c|}{ Identificação no caso } \\
\hline Organizacionais & $\begin{array}{l}\text { Novas formas de } \\
\text { estruturar as } \\
\text { organizações }\end{array}$ & $\begin{array}{l}\bullet \text { Mudança na missão da Itaipu } \\
\text { • Criação a Assessoria de Energias Renováveis } \\
\bullet \text { Criação do PTI } \\
\text { • Estruturação do Cibiogás }\end{array}$ \\
\hline Tecnológicas & $\begin{array}{l}\text { Desenvolvimento } \\
\text { tecnológico preventivo } \\
\text { ou curativo }\end{array}$ & $\begin{array}{l}\text { - Nacionalização das tecnologias; } \\
\text { Desenvolvimento dos biodigestores para realidade local; } \\
\text { - Pesquisas e aplicações de acordo com a realidade de cada } \\
\text { caso. }\end{array}$ \\
\hline Sociais & $\begin{array}{l}\text { Mudanças no } \\
\text { comportamento dos } \\
\text { consumidores }\end{array}$ & $\begin{array}{l}\text { - Difusão local do conhecimento; } \\
\text { - Mudança na percepção da comunidade local da viabilidade } \\
\text { e inclusão das tecnologias. }\end{array}$ \\
\hline Institucionais & $\begin{array}{l}\text { Redes locais, agências, } \\
\text { políticas, normas, } \\
\text { regras. }\end{array}$ & $\begin{array}{l}\text { Formação de alianças para pesquisa e desenvolvimento das } \\
\text { novas tecnologias; } \\
\text { Criação e aplicação das novas leis; } \\
\text { - Visibilidade do tema no governo e demais entidades de } \\
\text { classe. }\end{array}$ \\
\hline
\end{tabular}

Figura 2 - Ecoinovações desenvolvidas a partir do programa Cibiogás

Fonte: Elaboração própria

É importante destacar que, apesar das ecoinovações desenvolvidas a partir do desenvolvimento do nicho tecnológico, com apoio da Itaipu e da rede de parceiros, ainda não é possível observar uma mudança sociotécnica. Principalmente porque o processo encontra-se em difusão no contexto local de atuação com 11 unidades de produção que agora se espalham pelo Brasil (Vellinga, 2000). Esse entendimento também mostra que ainda há um regime tecnológico voltado para outras fontes de geração e distribuição de energia, mesmo que por fontes renováveis, mas a aceitação da comunidade local (ecoinovação social) e criação e aplicação de novas leis para geração e distribuição de energia acrescenta pontos de mudanças necessários para uma transição para a sustentabilidade.

\section{CONSIDERAÇÕES FINAIS}

Esse trabalho teve como objetivo analisar o processo de formação no nicho tecnológico do Centro Internacional de Estudos do Biogás na Itaipu Brasil, destacando os tipos de ecoinovações por ele desenvolvidas, por meio de uma abordagem multinível direcionada ao nicho tecnológico e ecoinovações por ele desenvolvidas.

O estudo identificou a importância de agentes "empreendedores" no processo de formação dos nichos, como a Itaipu, que atuaram, inicialmente, integrando em seu planejamento estratégico, os pilares da sustentabilidade, o que possibilitaram ações para beneficiar as comunidades locais e o estabelecimento de projetos nacionais. 
Assim, a formação do nicho está diretamente alinhada com a literatura sobre gestão estratégia dos nichos, por meio da experimentação e nacionalização de tecnologias. No caso da trajetória seguida pelo Cibiogás, nota-se uma busca pela nacionalização das tecnologias no sentido de adequá-las às necessidades locais, aos requisitos de cada um dos produtores e tornar essas tecnologias viáveis economicamente para a realidade local.

Os principais tipos de inovações foram identificados e relacionados com o conceito da ecoinovação debatido no referencial. No programa Cibiogás, foi possível identificar "ecoinovações organizacionais", por ser uma inovação surgida na Itaipu Brasil; "ecoinovações tecnológicas", principalmente devido ao desenvolvimento das tecnologias específicas para cada realidade dos projetos das unidades de demonstração e por utilizarem, muitas vezes, materiais reciclados na criação dos biodigestores; "ecoinovações sociais", relacionadas à difusão do conhecimento e da mudança de percepção da comunidade local sobre a aplicação e o uso da tecnologia; e "ecoinovações institucionais", uma vez que passaram a se desenvolver no nível meso de regulamentações e novos incentivos para geração distribuída de energia, transformando os biodigestores dos produtores e cooperativas de produtores em mini e microgeradores de energia.

O Programa do Cibiogás surgiu com objetivo de desenvolver tecnologias para soluções locais. Assim, o que se nota é a motivação inicial para a formação dos nichos e como o resultado do que foi desenvolvimento até o momento tem afetado a dimensão social. No caso do Cibiogás, como evidenciado pelos entrevistados, o programa passou a dar novas oportunidades para os pequenos produtores, tanto no sentido de melhoria ambiental no tratamento dos dejetos, quanto nas perspectivas social e econômica. Isto porque os produtores passaram a ser considerados mini ou microgeradores de energia e a economizar energia, já que passaram a consumir parte ou a totalidade necessária em seus processos produtivos.

A identificação quantitativa dos benefícios de adoção das soluções do programa Cibiogás por parte dos produtores, varia de maneira significativa, uma vez que cada projeto desenvolvido é único e voltado para as necessidades específicas do produtor. De maneira geral, o programa promove destinação adequada de resíduos, economia de energia elétrica e redução de despesas com produtos químicos, por meio da utilização de biofertilizantes oriundos do processo de biodigestão, que podem totalizar $30 \%$ das despesas totais do agricultor.

Assim, o que se percebe é a importância de iniciativas como estas para o processo de nacionalização de tecnologias para atender demandas e necessidades locais a fim de dar mais possibilidades de melhorias de qualidade de vida para comunidade e produtores no desenvolvimento dos seus negócios.

Do ponto de vista das contribuições, o trabalho se destaca pela aproximação realizada entre a perspectiva teórica dos nichos tecnológicos com a abordagem da ecoinovação, realizando, por meio da investigação de um caso de destaque nacional, a transposição de nichos tecnológicos para o desenvolvimento de inovações sustentáveis. Destaca-se, ainda, que contribuições práticas também emergiram por meio da sistematização de organização de uma rede de atores e seus papeis institucionais, bem como o relato de implementação do programa.

Como limitações, percebe-se que seria interessante expandir o estudo para outros nichos tecnológicos, seus processos de formação e os tipos de ecoinovações desenvolvidas para possíveis comparações e aprofundamento da aplicação da relação entre as abordagens da Gestão Estratégica dos Nichos e da ecoinovação. Outras possibilidades de pesquisas futuras recaem sobre a governança das relações entre os stakeholders envolvidos no processo de formação dos nichos e ainda a possibilidade de pesquisar de forma separada o contexto e atores sociais do processo de inovação dos nichos, com o objetivo de identificar a real participação dos atores no processo de desenvolvimento e aplicação da tecnologia e suas expectativas, evidenciando as principais percepções dos impactos e resultados alcançados.

\section{REFERÊNCIAS}


Andersen, M. M. (2008). Eco-innovation: towards a taxonomy and a theory. Conference entrepreneurship and innovation: organizations, institutions, systems and regions, Copenhagen, Denmark, 25, junho.

Arundel, A., \& Kemp, R. (2009). Measuring eco-innovation. (Unu-merit working paper series No. 2009-017). Netherlands: United Nations University.

Blackburn, W. R. (2007). The sustainability handbook: the complete management guide to achieving social, economic and environmental responsibility. Washington, DC: ELI Press.

Berkhout, F. (2002). Technological regimes, path dependency and the environment. Global Enviromental Change, 12, 1-4.

Berkhout, F. (2010). Technological regimes, environmental performance and innovation systems: tracing the links. In: Weber, M. \& Hemmelskamp, J. (Eds) Towards Environmental Innovation Systems. Berlin: Springer.

Bardin, L. (2011). Análise de conteúdo ( $3^{\mathrm{a}}$ reimpressão da $1^{\mathrm{a}}$ ed., Luís Antero Reto e Augusto Pinheiro, Trad.). Lisboa: Edições 70.

Bossle, M. B., Barcellos, M. D., Vieira, L. M. \& Sauvée, L. (2016). The drivers for adoption of ecoinnovation. Journal of Cleaner Production, 113, 861-872.

Caniëls, M. C. J. \& Romijn, H. A. (2008). Actor networks in strategic niche management: insights from social networks theory. Futures, 40, 613-629.

Carrillo-Hermosilla, J., Gonzalez, P. D. R. \& Konnola, T. (2009) Eco-innovation: when sustainability and competitiveness shake hands. New York: Palgrave Macmillan.

Cibiogás. (2019, março). Unidades de produção. Recuperado em 12 de março, 2019, de https://cibiogas.org/uds.

Denzin, N. K. \& Lincoln, Y. S. (2006). O planejamento da pesquisa qualitativa: teorias e abordagens. ( $2^{\mathrm{a}} \mathrm{ed}$, Sandra Regina Netz, Trad.). Porto Alegre: Artmed-Bookman.

Elzen, B. \& Wieczorek, A. (2005). Transitions towards sustainability through system innovation. Technological Forecasting \& social Change, 72, 651-661.

Foxon, T. \& Andersen, M. M. (2009). The greening of innovation systems for eco-innovation towards an evolutionary climate mitigation policy. Druid summer conference: innovation, strategy and knowledge, Copenhagen, Dinamarca.

Fussler, C., \& James, P. (1996). Driving eco-innovation: a breakthrough discipline for innovation and sustainability (1st ed.). London: Pitman Publishing.

Geels, F. W. (2002). Technological transitions as evolutionary reconfiguration processes: a multilevel perspective and a case-study. Research Policy, 31, 1257-1274.

Geels, F. W. (2004). From sectoral systems of innovation to socio-technical systems: insights about dynamics and change from sociology and institutional theory. Research Policy, 33(6-7), 897-920.

Geels, F. W. (2006). Co-evolutionary and multi-level dynamics in transitions: the transformation of aviation systems and the shift from propeller to turbojet (1930-1970). Technovation, 26(9), 9991016.

Geels, F. W. (2011). The multi-level perspective on sustainability transitions: responses to seven criticisms. Enviromental Innovation and Societal Transitions, 1, 24-40.

Geels, F. W. \& Kemp, R. (2007). Dynamics in socio-technical systems: typology of change processes and contrasting case studies. Technology in society, 29, 441-455. 
Formação de nichos tecnológicos e as ecoinovações: o caso do Cibiogás na Itaipu Brasil

Geels, F. W., \& Raven, R. (2006). Non-linearity and expectations in niche-development trajectories: ups and downs in Dutch biogas development (1973-2003). Technology Analysis \& Strategic Management, 18(3-4), 375-392.

Geels, F. W. \& Schot, J. (2007). Typology of sociotechnical transition pathways. Research Policy, 36, 399-417.

Genus, A., \& Coles, A. M. (2008). Rethinking the multi-level perspective of technological transitions. Research Policy, 37(9), 1436-1445.

Hermans, F., Van Apeldoorn, D., Stuiver, M., \& Kok, K. (2012). Niches and networks: explaining network evolution through niche formation processes. Research Policy, 42(3), 613-623.

Hermans, F., Stuiver, M, Beers, P. J. \& Kok, K. (2013). The distribution of roles and functions for upscaling and outscaling innovations in agricultural innovation systems. Agricultural Systems, 115, 117-128. https://doi.org/10.1016/j.agsy.2012.09.006.

Hess, D. J. (2016). The politics of niche-regime conflicts: distributed solar energy in the United States. Environmental Innovation and Societal Transitions, 19, 42-50.

Itaipu (2020, março). Geração. Recuperado em 19 março, 2020, de https://www.itaipu.gov.br/capaenergia

Itaipu. (2012). Projeto de Concretização: Cibiogás. Itaipu: Foz do Iguaçu.

Johnson, B., Edquist; C., \& Lundvall, B. A. (2003). Economic development and the national system of innovation Approach. First Globelics Conference, Rio de Janeiro, Brasil.

Kemp, R. \& Foxon, T. J. (2007, agosto). Typology of eco-innovation. In: Mei project: measuring eco-inovation. European Commission.

Kemp, R., \& Rotmans, J. (2010). The management of the co-evolution of technical, environmental and social systems. In: Weber, M. \& Hemmelskamp, J. (Eds) Towards Environmental Innovation Systems. 1(2), 33-55. Berlin: Springer.

Kemp, R., Schot, J., \& Hoogma, R. (1998). Regime shifts to sustainability through processes of niche formation: the approach of strategic niche management. Technology Analysis \& Strategic Management, 10(2), 175-195.

Kivimaa, P., Boon, W., Hyysalo, S. \& Klerkx, L. (2019). Towards a typology of intermediaries in sustainability transitions: a systematic review and a research agenda. Research Policy, 48, 10621075

Laak, W.W. M. Van Der., Raven, R. P. J. M., \& Verbong, G. P. J. (2007). Strategic niche management for biofuels: analyzing past experiments for developing new biofuel policies. Energy Policy, 35, 3213-3225.

Lopolito, A., Morone, P., \& Sisto, R. (2011). Innovation niches and socio-technical transition: a case study of bio-refinery production. Futures, 43(1), 27-38.

Markad, J., Raven, R., \& Truffer, B. (2012). Sustainability transitions: an emerging field of research and its prospects. Research Policy, 41(6), 955-967.

Mendonça, A.T. B. B. de; Cunha, S.K. da; Nascimento, T.C.(2018) relações multiníveis e inovação sustentável: o programa veículo elétrico da Itaipu Brasil. Revista Eletrônica de Ciência Administrativa, 17(3), 316-343 Set-Dez.

Merriam, S. B. (2009). Qualitative research: a guide to design and implementation. Califórnia: Jossey-Bass. 
Mylan, J., Morris, C., Beech, E. \& Geels, F. W. (2019). Rage against the regime: niche-regime interactions in the societal embedding of plant-based milk. Environmental Innovation and Societal Transitions, 31 .

Nelson, R. R. (2006a). As fontes do crescimento econômico. (Adriana Gomes de Freitas, Trad.). (Coleção Clássicos da Inovação). Campinas: Editora da Unicamp.

Nelson, R. R. (2006b). Economic development from the perspective of evolutionary economic theory. (Working Papers No. 2). Tallinn University of Technology, Tallinn, Estônia.

Raven, R. (2005, janeiro). Strategic niche management for biomass. Technical University EIndhoven. Recuperado em 15 agosto, 2017, de

https://www.researchgate.net/publication/257926311_Strategic_Niche_Management_for_biomass_ $\mathrm{PhD}$-Thesis .

Reid, A. \& Miedzinski, M. (2008, maio). Eco-innovation, final report for sectoral innovation watch. Brussels: Technopolis Group. Recuperado em 20 julho, 2017, de https://www.researchgate.net/publication/301520793_EcoInnovation_Final_Report_for_Sectoral_Innovation_Watch.

Rennings, K. (1998). Towards a theory and policy of eco-innovation: neoclassical and (co-) evolutionary perspectives (Discussion Paper No. 98-24). Mannheim, Germany: Centre for European Economic Research.

Safarzynska, K., Frenken, K., \& Van Den Bergh, J. C. J. M. (2012). Evolutionary theorizing and modeling of sustainability transitions. Research Policy, 41(6), 1011-1024.

Schot, J., \& Geels, F. W. (2008). Strategic niche management and sustainable innovation journeys: theory, findings, research agenda, and policy. Technology Analysis \& Strategic Management, 20(5), $537-554$.

Schumpeter, J. (1985). A Teoria do desenvolvimento econômico: uma investigação sobre lucros, capital, crédito, juro e o ciclo econômico. (Série Os Economistas, Maria Sílvia Possas, Trad). São Paulo: Abril Cultural.

Tamayo-Orbegozo, U., Vicente-Molina, M-A. \& Villarreal-Larrinaga, O. (2017). Eco-innovation strategic model. A multiple-case study from a highly eco-innovative European region. Journal of Cleaner Production, 142, 1347-1367.

Vellinga, P. (2000). Industrial transformation towards sustainability of the energy system. Integrated Assessment, 1(2), 157-163, 2000.

Walliman, N. (2006) Social Research Methods (1a ed.). London: Sage Publications.

Wittmayer, J. M., Avelino, F., Steenbergen, F. \& Loorbach, D. (2017). Actor roles in transition: insights from sociological perspectives. Environmental Innovation and Societal Transitions, 24, 4556.

Xavier, A. F., Naveiro, R. M., Aoussat, A., \& Reyes, T. (2018). Systematic literature review of ecoinnovation models: opportunities and recommendations for future research. Journal of Cleaner Production, 149, 1278-1302. 